\title{
HISTORIA DE LA DIDÁCTICA DE LAS CIENCIAS: UN CAMPO DE INVESTIGACION
}

\author{
Rómulo Gallego Badillo* \\ Adriana Patricia Gallego Torres* \\ Royman Pérez Miranda
}

\begin{abstract}
In this paper there will be given reasons about the necessecity of this new field of research and it will be suggested some delineations so as to separate it from the problems of which the didactics of the sciences, have been occupied up to the present.
\end{abstract}

\section{RESUMEN}

En el presente artículo se dan razones de la necesidad de este nuevo campo de investigación y se sugieren algunos delineamientos, y como tal, se separa de todos los problemas de los cuales los didactas de las ciencias experimentales se han ocupado hasta el presente.

Palabras claves: Didáctica, historia, fuentes, metodología

\section{INTRODUCCIÓN}

En las ciencias experimentales existe una tradición de estudios en esta área, cuyos primeros trabajos son historiográficos. Esta perspectiva cambió con las formulaciones de Los llamados epistemólogos historicistas principalmente, quienes propusieron una versión del desarrollo de los conocimientos científicos en términos de cambios paradigmáticos (kuhn, 1972) o de la emergencia de programas de investigación (Lakatos. 1983) Con este autor surgió la indispensable realización de una historia interna y de una externa imbricadas. T.S. Kuhn e I. Lakatos enseñaron que la historia de las ciencias no podía ser asumida desde una visión lineal y acumulativa, ni como fruto de genialidades aisladas y descontextualizadas. Recuérdese que por fuera de la categoría de comunidad científica, S. Toulmin (1972), anotó, desde su mirada evolucionista, que ese desarrollo obedecía a una genealogía de maestros y discípulos, de profesores y alumnos.

En cuanto a la didáctica de las ciencias experimentales, se ha puntualizado que es una disciplina teóricamente fundamentada (Gil y Colab. 1999), en la que sus campos de conocimiento han sido delimitados (Gil, Carrascosa y Martínez, Terrades, 1999). Aun cuando hay críticas en relación con estas afirmaciones (Marín, 1999), se habla ya de que se cuenta con una ciencia de enseñar ciencias (Izquierdo y Sanmarti, 2001). En consecuencia, una demostración de tal aserto ha de venir, necesariamente, de una historia de esa didáctica desde una mirada estrictamente investigativa. A partir de la conciencia de que una cosa es la historia de las ciencias experimentales y otra la de la enseñanza de las mismas, como un ámbito relativamente autónomo de la anterior, se sostiene aquí que

\footnotetext{
*Profesor U.P.N.

${ }_{* \star \star}^{* \star}$ Universidad de Valencia España, Departamento de Didáctica de las Ciencias Experimentales y Sociales.

${ }^{* * *}$ Profesor U.P.N.
} 
lo establecido por esos epistemólogos historicistas, se conforma en una perspectiva conceptual y metodológica para adelantar dicho nuevo campo de investigación.

\section{LA HISTORIA DE LA DIDÁCTICA}

Hacer la historia de la didáctica de las ciencias experimentales, es dar cuenta documentada de los procesos de Construcción de la misma, como una disciplina científica, desde sus propuestas pre-paradigmáticas hasta el presente, cuando se piensa, como ya se dijo, que es una disciplina científica. Esa historia ha de explicitar las maneras de cómo hoy la comunidad de especialistas ha llegado a las elaboraciones conceptuales y metodológicas que compiten entre si, como también a la concepción dominante, es decir, la que mejor resuelve los problemas de la enseñanza o la que cuenta con más apoyo empírico, entre todas.

Si se admite lo afirmado, hay que pensar, entonces, que se hace también indispensable examinar esos cuerpos conceptuales y metodológicos, a partir de un punto de vista epistemológico y en este sentido, concebir tal didáctica como una actividad, esto es, aquella que adelantan los investigadores en la enseñanza de las ciencias dentro de sus programas de investigación. Aclárese que al hacer referencia a la actividad se está diciendo, para reiterarlo, que ella obedece a fundamentaciones teóricas.

Hacer historia de la didáctica de las ciencias experimentales, implica abandonar el paradigma habitual que la reduce a una dimensión completamente instrumental, algorítmica, para centrarse en la convicción de que a partir del momento en que la enseñanza de las ciencias se constituyó en un derecho y hasta el presente cuando se han construido modelos educativos de las mismas, los cuales hablan en favor de la existencia de unas concepciones didácticas que es preciso develar. En este sentido es menester puntualizar en que una cosa ha sido y es la actividad de los científicos profesionales y otra la desplegada y que se lleva a cabo en las instituciones educativas, por cuanto la ciencia escolar es una versión basada en la didactización, tanto de las elaboraciones como de las actividades de los especialistas en la producción de conocimiento científico.

Este proceso de transformación, de reorganización y de reelaboración que los especialistas en educación matemática denominan transposición didáctica (Kang y Kilpatrick, 1992), ese hacer de las ciencias propio de los profesionales de ellas, las de las instituciones escolares, sin tergiversaciones en principio, lo que constituye la base para elaborar La historia que aquí se propone, en La medida en que supone unas concepciones por precisar, a partir de las cuales esa didactización ha sido llevada a cabo. Esto podría constituirse en la historia interna, la cual tendría que ser complementada indispensablemente con la externa, que tendría que ver con la dominancia de ideologías específicas y las intencionalidades de proyectos culturales, sociales, políticos y económicos tipificables que determinan o han decidido qué versión de ciencia enseñar.

\section{DELIMITACIONES}

La indagación histórica ha de comenzar por fijar unos períodos específicos, lo que constituye de por sí un problema en razón de que el fundamento podría ser determinado por las diferentes revoluciones científicas, en cada una de las disciplinas. Son, por consiguiente, los investigadores en la didáctica de cada una de las ciencias experimentales, quienes han estudiado la historia de sus áreas de trabajo, los llamados a 
fijar esos períodos e incluso les corresponde a estos especialistas establecer los criterios que consideren necesarios para cada uno de los correspondientes cambios paradigmáticos en los planteamientos de la enseñanza de los mimos y de las ciencias, en general. De la misma manera, el tipo de transformaciones epistemológicas que implícita o explícitamente pudieron conducir o no a nuevos esquemas acerca del carácter de la didáctica.

Anótese, que únicamente en casos extraordinarios y excepcionales, como en los niveles educativos de doctorado y posdoctorado por lo general, los profesores de ciencia enseñan contenidos en los que las respectivas comunidades de especialistas no están produciendo nuevos conocimientos ni tecnofactos (Gallego, 1997), es decir, los marcos conceptuales y metodológicos de sus investigaciones, han superado aquello que en las instituciones educativas es objeto de estudio. Se puede afirmar, con T. S. Kuhn (1972), que lo que se hace es historia de las ciencias, no desde la perspectiva metodológica de los historiadores, sino desde los parámetros de una concepción didáctica específica.

En otras palabras, la didáctica de las ciencias experimentales ha de dar cuenta de Las razones educativas por las cuales en las instituciones escolares se hacen objeto de enseñanza y de estudio paradigmas o programas de investigación científica que históricamente fueron base para reformulaciones o cambios teóricos. Habría entonces que examinar si esos contenidos por enseñar, desde la transposición didáctica respectiva, les permiten al estudiantado reflexionar en torno a los problemas que resolvió y de aquellos que no pudo solucionar, por lo que se hicieron necesarias reconceptualizaciones o cambios teóricos fundamentales.

Otra opción, dado que el planteamiento rector es el de la reestructuración de la ciencia que producen Las comunidades científicas, para hacerla objeto de enseñanza, parece ser claro que la delimitación de los períodos históricos debería provenir de las revoluciones en las ideas sobre la naturaleza de la didáctica de las ciencias experimentales, aun cuando tales períodos fueran sólo dos: El de la etapa pre-científica de esta disciplina y la de su constitución como un ámbito de trabajo teóricamente fundamentado. Se trata de hacer historia de la didáctica, desde sus desarrollos teóricos internos independizándola relativamente de los cambios acaecidos en las ciencias experimentales.

De acuerdo con lo anterior, podrían tipificarse otros períodos a partir de la precisión de los enfoques concretos de enseñanza que han dominado en diferentes momentos. Así, se podría estipular una época dominada por la reducción de lo elaborado por los científicos a definiciones de conceptos y demostraciones magistrales por parte de los docentes, caso este en el que domina y se exige la actitud pasiva de los discentes. Otra podría ser aquella en la que el docente hace explicaciones magistrales, seguidas de prácticas de laboratorio, reguladas por el seguimiento de guías-recetas. Finalmente, una tercera, en la que los estudiantes son los constructores de su propio conocimiento, bajo la asesoría permanente del didacta. Cada una de esta épocas obedece a una concepción sobre las ciencias y su enseñanza.

Otro punto de vista por considerar, sería aquel en el cual, si bien se enclava en la psicología de la educación o en las actuales teorías sobre la cognición, los períodos podrían ser fijados desde la dominancia de las clases de aprendizajes que en cada momento se ha esperado que el estudiantado haga. El memorístico y repetitivo discriminando en este caso, por ejemplo, la presencia del behaviorismo y las aproximaciones empiropositivistas. De manera correspondiente, dada la relación que 
siempre se ha admitido, de una enseñanza por objetivos, según la racionalidad instaurada por el taylorismofordismo.

Dentro de la mirada construtivista, otro período sería el establecido por el aprendizaje de tipo piagetano, con el cual se tendría una enseñanza por logros, siempre y cuando lo actitudinal estuviera teóricamente presente. Esa enseñanza, más que centrada en la repetición de contenidos curriculares, perseguiría la construcción por parte del alumnado, de actitudes positivas hacia las ciencias (Schibecci, 1984), siguiendo fundamentaciones teóricas rigurosas (Furió y Vilches, 1997). Se trataría de perseguir un aprendizaje signado por la dialéctica de La asimilación-acomodación. Habría que examinar el hecho incuestionable de si sí o no esta formulación ha tenido o no una influencia significativa en la transposición didáctica.

Posicionados los investigadores en la historia de la didáctica de las ciencias experimentales, el análisis podría hacerse desde la propuesta del aprendizaje significativo (Ausubel, Novaky Hanesian, 1983; Moreira, 1990), regulado por las estrategias de elaboración de mapas conceptuales y de la Ve heurística (Novak y Gowin, 1984). Tal puntualización debería ser uno de los fundamentos de la reestructuración didáctica de las diferentes ciencias experimentales, ya que introduce una concepción innovadora de la enseñanza y, por consiguiente, sería asumible como un paradigma didáctico de otro tipo. Habría que estudiar si esto se ha dado o no.

Otro punto de partida estaría dado por el de la formulación del aprendizaje como cambio conceptual y metodológico (Gil y Carrascosa, 1985), en el que se postula que todos los conceptos de las ciencias experimentales son metodológicos, a la vez que todas las estrategias metodológicas, son conceptuales. Más allá de esta visión restrictiva, hay detrás de tal propuesta de aprendizaje la convicción de que las teorías de las ciencias experimentales son hipotético-deductivas, es decir, son sistemas de formulación de soluciones lógicas y experimentales de las mismas. Hay aquí también un paradigma didáctico que posibilita reestructurar de otra manera una ciencia dada, para hacerla objeto de enseñanza. Siguiendo este paradigma la didactización tendría que hacerse desde un enfoque investigativo.

Vendrían ahora planteamientos que pueden ser considerados complementarios. El aprendizaje como cambio conceptual, metodológico y actitudinal (Gené, 1991) y el del cambio conceptual, metodológico, actitudinal y axiológico (Gallego y Pérez, 1994). El primero obliga a que la reestructuración didáctica se realice evidenciando las miradas actitudinales que han estado detrás de toda actividad (Izquierdo, 2000) característica de las comunidades científicas. El segundo imbrica los problemas en relación con los principios éticos (Echavarría, 1955) que han guiado y dirigen la producción de conocimientos científicos. La cuestión axiológica ha de ser pensada, para la transposición didáctica correspondiente, desde la perspectiva del impacto que las ciencias tienen en la estructura de valoraciones de cada comunidad a sociedad.

No obstante lo anterior y no agotada la temática, podría pensarse en que los nuevos investigadores en la didáctica de las ciencias experimentales habrían de considerar otros criterios para delimitar los períodos desde los fundamentos mismos de sus proyectos de trabajo. De cualquier manera se espera que se conforme dicha comunidad en este campo para que sea ella la que regule la admisibilidad de tales criterios. 


\section{DE LAS FUENTES}

En principio, si el punto de partida para hacer la historia de la didáctica de las ciencias experimentales son las concepciones de enseñanza y de aprendizaje que han direccionado las transposiciones didácticas de los cuerpos conceptuales y metodológicos producidos por las respectivas comunidades científicas, en los períodos que establezcan como necesarios los especialistas en este nuevo campo de conocimiento, las fuentes escritas de primera mano han de ser las revistas en las que se publican las investigaciones sobre la enseñanza de las ciencias.

Aun cuando estas revistas surgen y se consolidan a partir de la segunda mitad del Siglo $X X$., es en ellas en donde han de estudiarse las series de paradigmas que han dominado, tanto como las revoluciones paradigmáticas que en las concepciones sobre la enseñanza de dichas ciencias se han sucedido. Al respecto se tendría que identificar la influencia que tales revistas habrían tenido en la práctica habitual de los profesores de ciencia en ejercicio (Alíberas. Gutiérrez e Izquierdo, 1989).

Otra fuente escrita es la suministrada por los textos escolares, en cuanto a que es en ellos en donde se expresan las concepciones didácticas y epistemológicas sus autores. Un departamento universitario dedicado a esta disciplina, en consecuencia, ha de contar en su biblioteca con una sección dedicada a recuperar, conservar y clasificar eL mayor número de dichos textos pasados y actuales, en todos los niveles de ayer y hoy de los diferentes sistemas educativos nacionales e internacionales.

Una biblioteca con una sección de tales características, daría pie para crear la subsección de historia comparada de la didáctica de las ciencias experimentales, sobre todo si esta se enfoca desde la historia externa, es decir, de la influencia de los distintos sistemas ideológico políticos que han privilegiado e impuesto concepciones caracterizables sobre estas ciencias y su enseñanza, a la vez que obstaculizado el desarrollo de una didáctica como disciplina teóricamente fundamentada, conceptual y metodológicamente autónoma, de manera relativa, al mismo tiempo que sostenido el paradigma instrumental.

Anótese que la apertura de este nuevo campo de investigación dentro de la didáctica de la ciencias experimentales, exige la estimación de los viejos y olvidados textos escolares, aun cuando no recojan en sus páginas, según sus fechas de publicación, Los nuevos paradigmas científicos, dado que mayor valor histórico poseen, por lo que se constituyen en verdaderas reliquias para los historiadores del pensamiento didáctico.

Los textos escolares se constituyen en fuentes, precisamente porque a partir del examen de la organización de los contenidos que presentan, permiten inferir la reconstrucción histórica que hacen del desarrollo de la ciencia que ponen a disposición de la sociedad, en general, el orden didáctico que instauran, la concepción de la respectiva ciencia que imponen a los enseñantes, como también el sometimiento de estos a un tipo de trabajo profesional con el alumnado, por fuera del cual tal profesionalidad se hace objeto de dudas y de sospechas. Dígase aquí, que resultaría sorprendente una transposición didáctica, según las aproximaciones constructivistas del aprendizaje, basada en textos escolares en los cuales las versiones no se hayan hecho desde algunas de estas perspectivas. Se sostiene que ningún docente puede hacer una enseñanza de las ciencias, sin examinar rigurosamente los textos escolares que ofrece el mercado bibliográfico, ya que de otra manera su profesionalidad estaría en discusión (San martí, 2000). 
Si habitualmente o por formación los docentes de cada una de las ciencias experimentales y sus disciplinas se han guiado siempre por textos didácticos, hay que decir, con el beneficio de la duda, que han enseñado versiones didactizadas, que se han sometido, sin quererlo o no, a determinadas aproximaciones epistemológicas y, como ya se dijo, a una reelaboración histórica de la ciencia que enseñan, que no obedece a la dinámica histórica de su desarrollo conceptual y metodológico interno. Tal inscripción, conciente o no, es la que podría hallarse en la base de la admisión generalizada de que las ciencias experimentales no han obedecido a transformaciones sociales, culturales, políticas y económicas de aquellas sociedades en las cuales el desarrollo científico endógeno fue posible.

Por supuesto que, en rigor histórico, otra fuente para el estudio la constituyen los profesores de ciencia pensionados, jubilados o eméritos que aún siguen vivos. Sus ideas sobre la enseñanza y las formas como la ejercieron en los correspondientes niveles de los sistemas educativos en los cuales se desempeñaron., deben ser recogidas, catalogadas, analizadas y sistematizadas, ya que constituyen un testimonio, también valioso, para la historia de la didáctica de estas ciencias. Dicha fuente adquiere sentido si a los entrevistados se les solícita cuáles fueron los principales textos didácticos a partir de Los duales planificaron su trabajo de enseñanza y de los mandamientos oficiales a los cuales, del una manera u otra, se sometieron. En este orden metodológico, cuales fueron sus posiciones alternativas en relación con la didáctica de las ciencias que enseñaron.

Una cuarta fuente serían los registros de los planes de estudio o de los proyectos curriculares que se conservan en las dependencias oficiales de los ministerios del ramo, por cuanto en los respectivos documentos se consignan, no sólo apreciaciones de lo que debe enseñarse, sino las intencionalidades mediante las cuales las ciencias experimentales se hicieran objeto de enseñanza y los objetivos que se perseguían con cada una de elLas en Los respectivos niveles del correspondiente sistema educativo de cada época. Incluso, se hace menester rastrear el establecimiento de propuestas y secuencias temáticas sugeridas o impuestas, por cuanto ellas reflejan reestructuraciones $\mathrm{y}$, por tanto, concepciones acerca de una didáctica oficial.

\section{UNA PROPUESTA METODOLÓGICA}

Si el estudio histórico se centra en el análisis de los textos y en el registro de Los planes de estudio y proyectos curriculares oficiales, es de esperarse que los investigadores conozcan la historia de la ciencia correspondiente, los libros y las revistas en Los que se propusieron los nuevos paradigmas, para efectos de comprender cual fue la transposición didáctica que se hizo, como también la reorganización de los contenidos, centrándose o no ella en el estricto orden histórico.

Por lo demás, esos investigadores, también han de tomar como opción metodológica el análisis hermenéutico, con miras a detectar las concepciones epistemológicas y didácticas que se hallan en aquellos textos escolares o en los planes y proyectos curriculares que, por Lo general, no lo explicitan, con todo el riesgo profesional que ello conlleva. Surge en consecuencia, la necesidad de entrevistar a los autores de los textos, de ser posible, con miras a contrastar las conclusiones a las que el análisis de tales textos han dado lugar.

El punto de partida es eminentemente interpretativo, lo que significa sin lugar a dudas, el predominio de una investigación de tipo cualitativo. Sin embargo, puede ser ella 
complementada con análisis cuantitativos, sobre todo, en el orden de la comparación del número de unidades didácticas, tanto de los textos como de los planes y proyectos curriculares y el predominio de tratamientos de temáticas particulares durante períodos determinados.

\section{CONCLUSIONES}

Es un campo nuevo que se propone y, aun cuando no se niega que se encuentren algunos estudios al respecto (Gil, Carrascosa y Martínez Terrades, 1999), lo cierto es que este campo está por hacerse y por consiguiente la creación de una comunidad de especialistas. Las investigaciones que han de venir reconceptualizarán los fundamentos que aquí se presentan, a la vez que mejorarán los delineamientos metodológicos.

Se quiere insistir en que la historia de la didáctica de las ciencias experimentales constituirá el espacio de rigor que complementará las demostraciones de que esta se ha conformado hoy como una disciplina científica, después de diferentes fundamentaciones ya abandonadas, cuyas críticas hicieron posibles los diferentes paradigmas que les sucedieron.

La tarea es ardua, por lo que sería recomendable abocar el trabajo desglosándolo en unidades didácticas para cada una de las ciencias que se han hecho objeto de enseñanza, sin perder de vista que para cada período que se pretenda historiar, el orden de los libros de texto correspondiente a ese período puede constituirse en una guía.

\section{BIBLIOGRAFIA}

ALIBERAS, J., GUTIÉRREZ, R. e IZQUIERDO, M. 1989. La didáctica de las ciencias: Una empresa racional. Enseñanza de las Ciencias, 7(3), 227 - 280

AUSUBEL, D., NOVAK, J. D. y HANESIAN, H. 1983. Psicología Educativa. Un punto de vista Cognoscitivo. Segunda Edición. México, Trillas.

ECHEVARRÍA, J.1955. Filosofía de las ciencias. Madrid, Akal.

FURIO MAS, C. y VILCHES, A. 1997. La dimensión afectiva del aprendizaje de las ciencias: actitudes hacia las ciencias y relaciones ciencia, tecnología y sociedad. En: La enseñanza-aprendizaje de las ciencias de la naturaleza en la educación secundaria. Barcelona, CE. Universidad de Barcelona.

GALLEGO BADILLO, R. y PEREZ MIRANDA, R. 1994. Representaciones y Conceptos científicos. Un programa de investigación. Santafé de Bogotá, Universidad Pedagógica Nacional, Departamento de Química.

GALLEGO. BADILLO, R. 1997. discurso constructivista sobre las tecnologías. Santafé de Bogotá, Cooperativa Editorial Magisterio..

GENE, A.1991. Cambio conceptual y metodológico en la enseñanza y el aprendizaje de la evolución de los seres vivos. Un ejemplo concreto. Enseñanza de las Ciencias, 9(1), 26-27. 
GIL PEREZ, D. y COLAB. 1999 ¿Puede hablarse de consenso constructivista en la educación científica? Enseñanza de la Ciencias, 17(3), 503-512.

GIL PEREZ, D. y CARRASCOSA ALLIS, J. 1985. Science learning as conceptual and methodological change. Eur. J. Educ., 7(3), 231-253.

GIL PÉREZ, D., CARRASCOSA ALLIS, J. Y MARTíNEZ TERRADES, F. 1999. El surgimiento de la didáctica de las ciencias como campo específico de conocimientos. Revista Educación y Pedagogía. Vol XI No. 25, 13-65

IZQUIERDO, M y SANMARTI, N. 2001. Hablar y escribir para enseñar ciencias. Enseñanza de las Ciencias, Número Extra. VI Congreso.

IZQUIERDO, M. 2000. Fundamentos epistemológicos. En: Didáctica de las ciencias experimentales, 35 - 64. Alcoy (España), Marfil.

KANG, W. AND KILPATRICK, J. 1992. Didactic transposition in mathematics textbooks. For de learning of mathematics, 12(1), 2-7.

KUHN, T. S. 1972, La estructurado las revoluciones científicas. México, Fondo de Cultura Económica.

LAKATOS I. 1983. La metodología de los programas de investigación científica. Madrid, Alianza.

MARIN, N. 1999. Delimitando el campo de aplicación del cambio conceptual. Enseñanza de las Ciencias, 17(1), 80-92

MOREIRA M. A. 1990. Pesquisa em ensino: O Ve epistemológico de Gowin. Sao Paulo, Editora Pedagógica Universitaria Ltda.

NOVAK J. D. Y GOWIN, B. 1984 Learning how to learn. Cambridge. University Press.

SANMARTI, N. 2000. El diseño de unidades didácticas. En: Didáctica de las Ciencias Experimentales. Alcoy (España), Marfil.

SCHIBECCI, R. A. 1984. Attitude to science an update. Studies in science education, 11: 26-59.

TOULMIN, S. 1972. La comprensión humana. Vol. 1. El uso colectivo y la evolución de los conceptos. Madrid, Alianza. 